TITLE:

\title{
Acute effect of electrical stimulation on the infraspinatus muscle using different types of muscle contractions and shoulder joint positions
}

\section{AUTHOR(S):}

Yanase, Ko; Hasegawa, Satoshi; Nakamura, Masatoshi; Tanaka, Hiroki; Umehara, Jun; Araki, Kojiro; Ibuki, Satoko; Ichihashi, Noriaki

\section{CITATION:}

Yanase, Ko ... [et al]. Acute effect of electrical stimulation on the infraspinatus muscle using different types of muscle contractions and shoulder joint positions. Gazzetta Medica Italiana Archivio per le Scienze Mediche 2017, 176: 647-654

\section{ISSUE DATE:}

2017-12-01

URL:

http://hdl.handle.net/2433/243888

\section{RIGHT:}

C 2017 EDIZIONI MINERVA MEDICA; 許諾条件に基づいて掲載していま す。 
Acute Effect of Electrical Stimulation on the Infraspinatus Muscle using Different Types of Muscle Contractions and Shoulder Joint Positions

Ko Yanase ${ }^{{ }^{*}}$, Satoshi Hasegawa ${ }^{1}$, Masatoshi Nakamura ${ }^{2}$, Hiroki Tanaka ${ }^{3}$, Jun Umehara ${ }^{1}$, Kojiro Araki $^{4}$, Satoko Ibuki ${ }^{1}$, Noriaki Ichihashi ${ }^{1}$

${ }^{1}$ Human Health Sciences, Graduate School of Medicine, Kyoto University, Kyoto, Japan; ${ }^{2}$ Institute for Human Movement and Medical Sciences, Niigata University of Health and Welfare, Niigata, Japan; ${ }^{3}$ Unit of Rehabilitation, Kyoto University Hospital, Kyoto, Japan; ${ }^{4}$ Department of Rehabilitation, Sapporo Tokushukai Hospital, Sapporo City, Japan

* Corresponding author: Ko Yanase, Human Health Sciences, Graduate School of Medicine, Kyoto University, 53 Shogoin-Kawahara-cho, Sakyo-ku, 606-8507, Kyoto, Japan.

E-mail: kou.yanase1991@gmail.com 


\section{ABSTRACT}

BACKGROUND: Electrical stimulation (ES) is considered to be effective on infraspinatus muscle with functional decline and atrophy. However, it is not clear which parameters of ES e.g., types of muscle contractions and shoulder joint positions have good effects for inducing hypertrophy and increasing muscular strength. The purpose of this study was to determine the acute effects of ES in different types of muscle contractions and shoulder joint positions on the infraspinatus muscle by measuring the muscle swelling after ES.

METHODS: Forty subjects were randomly assigned to one of five groups: an isometric contraction with maximum internal rotation (IR), an isometric contraction with neutral position of rotation (NEUT), an isometric contraction with maximum external rotation (ER), a concentric contraction (CONCEN), and a control (CON) group. Subjects in all groups except for the CON group received ES for 20 min. The CON group did not receive intervention. The muscle thicknesses of the superior and inferior infraspinatus were measured using ultrasonography before and immediately after a single ES intervention.

RESULTS: Percentage change in muscle thickness of the inferior infraspinatus was greater in the IR and NEUT groups than the muscle thickness of the CON group; however, the muscle thickness of the superior infraspinatus did not differ significantly among the groups.

CONCLUSIONS: The results of this study indicate that applying ES to the inferior infraspinatus muscle in an isometric contraction with the muscle in a stretched position is an effective method to induce greater muscle swelling.

Key words: Electrical stimulation, Rotator cuff, Muscle contraction, Shoulder joint 
Introduction

The infraspinatus muscle is one of the rotator cuff muscles involved in the external rotation of shoulder, and it plays an important role in the dynamic and static stability of the shoulder.

Numerous studies have reported that functional decline and atrophy of the infraspinatus muscle occur in athletes who participate in overhead sports such as tennis or volleyball ${ }^{1-5}$. Thus, atrophy of the infraspinatus muscle is a serious problem with overhead athletes. An adequate training regime on the infraspinatus muscle is required for increasing muscle mass and strength.

Electrical stimulation (ES) is a widely used training method for increasing muscle strength. Several studies have reported that ES training increased the strength of the lower limb muscles and improved their performance in healthy persons and athletes ${ }^{6-10}$, and it is considered to be effective for enhancing muscle function of the infraspinatus muscle. Previous studies have been conducted to estimate which parameters, e.g., intensity, frequency, and time are effective in increasing strength ${ }^{11-}$ ${ }^{14}$. Filipovic et al. ${ }^{13}$ reported that muscle strength was significantly increased after an ES application for both contraction types, i.e., concentric and isometric contraction, using ES. Moreover, the systematic review reported that in order to increase maximum isometric voluntary contraction, using ES with an isometric contraction is recommended. Likewise in order to increase dynamic muscle strength such as maximum isokinetic strength and concentric contraction, ES with an concentric contraction is recommended ${ }^{13}$. However, this systematic review could not accurately compare the differences between the effects of isometric and concentric contraction using ES because the study did not standardize other parameters except for types of muscle contractions, such as the current intensity, electrode, device, frequency, number of subjects, and time of stimulation. Therefore, it is necessary to determine the effective muscle contraction type, isometric or concentric contraction using standardized conditions and parameters during ES intervention.

On the other hand, one study focused on the effect of the joint position during ES with an isometric contraction ${ }^{15}$. Fahey et al. ${ }^{15}$ compared the effect of six weeks of ES intervention in 
isometric contraction with the knee flexed versus the extended position. The results showed a larger increase in muscle strength in the knee flexed group than the extended group. The study suggested that the quadriceps were stretched during ES in the knee flexed group, which may have caused greater tension during ES for the knee flexed group than the extended group. The greater tension of the flexed group during ES could have resulted in the superior muscle strengthening. Therefore, it is possible that performing ES with the infraspinatus muscle in a lengthened position could be more effective than in the shortened position.

Acute change in muscle thickness is known to occur following strength training ${ }^{16,17}$. This immediate change in thickness is considered to be due to acute cell swelling which leads to an increase in protein synthesis and a decrease in proteolysis ${ }^{16}$. Also, the increased osmotic pressure may lead to the increase in muscle size, and it may indirectly signal satellite cells to proliferate. ${ }^{18}$ Therefore, an immediate increase in muscle thickness following strength training was used as an index of muscle swelling in previous studies ${ }^{16,17,19}$. In addition, Farup et al. ${ }^{17}$ examined the effect of strength training on the acute response in muscle thickness after a single strength training session and the hypertrophic response after 6 weeks of strength training. They found that an immediate increase in muscle thickness occurred following strength training, and it gradually returned to the baseline after $48 \mathrm{~h}$. A hypertrophic response was also found following the 6-week intervention period. Therefore, it is considered rational to measure the immediate response in muscle thickness to evaluate the effect of training.

The purpose of this study was to determine the acute effects of ES on the infraspinatus muscle thickness using different types of muscle contraction: isometric or concentric. We also investigated using different positions of the shoulder joint: maximum internal rotation, neutral rotation, or maximum external rotation. Muscle thickness, as an index of muscle swelling, was examined and compared before and after the ES intervention in this study. Therefore, we investigated the degree of acute effects of ES on the infraspinatus muscle thickness for estimating the effectiveness of training. The hypothesis in this study is that ES in positions with the 
infraspinatus muscle in a stretched position would induce greater muscle swelling compared to ES with the muscle in other positions as an acute effect.

Materials and methods

Subjects

Forty healthy men with the following characteristics: an age of $24.4 \pm 3.6$ years, a body height of $172.1 \pm 5.6 \mathrm{~cm}$, and a body mass of $67.3 \pm 7.6 \mathrm{~kg}$, participated in this study. The right or left shoulder of each subject was randomly selected. We calculated the sample size needed for a oneway analysis of variance (ANOVA) with an effect size of 0.6 , an $\alpha$ error of 0.05 , and a power of 0.8 using a $\mathrm{G}^{*}$ power 3.1 software program (Heinrich Heine University, Duesseldorf, Germany). Subjects who had previous or current upper-arm injuries and those who performed upper limb resistance trainings more than once per week were excluded from the study. All subjects were randomly distributed into five groups of eight people. The five groups were a control (CON), an isometric contraction with maximum internal rotation (IR), an isometric contraction with neutral rotation (NEUT), an isometric contraction with maximum external rotation (ER), and a concentric contraction (CONCEN) group. This study was approved by the ethics committee of Kyoto University Graduate School and the Faculty of Medicine (E-1994). Each participant gave informed consent before taking part in the study.

\section{Experimental design}

The muscle thickness of the infraspinatus muscle, for evaluating muscle swelling, was measured before the ES application. Then, one 20 min session of ES was applied to all groups except for the CON group; the CON group received no intervention and remained rested for $20 \mathrm{~min}$. The muscle thickness of the infraspinatus was assessed within 5 min after the ES application in the intervention group, and after 20 min of rest in the CON group. All measurements were conducted at Kyoto University. 


\section{Muscle thickness}

The muscle thickness of the superior and inferior infraspinatus was measured using a B-mode ultrasound device (LOGIQ e, General Electric, Duluth, GA, USA) with an $8 \mathrm{MHz}$ linear-array probe and a gain of $58 \mathrm{~dB}$. The subjects were in the prone position on the treatment table with their arms at the side of their body.

The measurement locations of the superior and inferior infraspinatus are presented in Figure 1. The measurement site for the superior infraspinatus was defined as the upper third of the infraspinatus muscle width and the medial fourth of the infraspinatus length. The measurement for the inferior infraspinatus was obtained at the lower third of the width and medial fourth of the length of the muscle of the muscle. The width of the muscle was defined as the distance from the spina scapulae to the upper border of the teres major, and the length of the muscle was defined as the distance from the medial edge of the scapula to the lateral edge of the acromion. The measurements were obtained twice, and their mean values were used for further analysis. The baseline test-retest intraclass correlation coefficient (ICC) value for the muscle thickness of the superior and inferior infraspinatus was excellent (ICC > 0.99). In addition, the percentage change of muscle thickness was calculated using the following formula: [(final measurement - initial measurement)/initial measurement] $\times 100$.

\section{Procedures}

Subjects in all groups, except the CON group, received ES on the infraspinatus muscle using an ES device (Homer Ion Co. Ltd., Tokyo, Japan). The subjects lay in the prone position with their arms abducted at $90^{\circ}$ and elbows flexed at $90^{\circ}$. The forearm was placed on the edge of the bed. Before the electrodes were attached to the skin, the surface of the skin was cleaned to reduce skin resistance using a cotton tissue soaked in alcohol. Bipolar electrodes, $5 \mathrm{~cm} \times 5 \mathrm{~cm}$, were used. While one electrode was attached to the skin over the motor point of the infraspinatus muscle, the 
other was placed over the muscle belly. The exponential climbing pulse was used in this experiment $^{20}$. We used the following ES parameters: a $250 \mu$ s pulse width, $20 \mathrm{~Hz}$ frequency, and $5 \mathrm{~s}$ stimulation including a 2 s pause duty cycle. During the initial five min of muscle stimulation, the intensity was gradually increased to the maximum level that each subject could tolerate, and the intensity was sustained for the following 15 min. The current intensity average plus or minus the standard deviation was $84.6 \mathrm{~mA} \pm 22.1 \mathrm{~mA}$. The pain was measured using the $100 \mathrm{~mm}$ visual analog scale (VAS) at the fifth minute from the starting time of ES.

Differences between joint positions and muscle contractions

We conducted ES on all groups, except for the CON group. In the IR, NEUT, and ER groups, the arms were fixed using a belt so that no joint movement would occur owing to stimulation, i.e., isometric contraction. The shoulder joint was abducted at $90^{\circ}$, with the elbow flexed at $90^{\circ}$. Furthermore, shoulder rotation was adjusted by controlling the belt for each group: a maximum internal rotation for the IR group, a neutral rotation for the NEUT group, and a maximum external rotation for the ER group (Figure 2). Maximum internal and external rotation of the shoulder was defined as the maximum angle that neither caused pain nor made the subjects feel uncomfortable. The IR group maintained $82.5^{\circ} \pm 9.6^{\circ}$ of internal rotation and the ER group maintained $86.1^{\circ} \pm$ $13.5^{\circ}$ of external rotation. In contrast, the arm was not fixed in the CONCEN group permitting concentric muscle contraction during ES (Figure 2). The average joint motion due to concentric contraction during ES was $45.7^{\circ} \pm 12.1^{\circ}$ from drooping position to the direction of external rotation.

\section{Statistical analysis}

The data were analyzed using SPSS (version 22.0J, IBM, Tokyo, Japan) at Kyoto University. First, the age, body height, body mass, current intensity, VAS, muscle thickness, and the percentage change of the muscle thickness were assessed using a Shapiro-Wilk test. Paired t-tests were used for the muscle thickness before and after the ES application. In addition, these normally distributed 
measurements except for muscle thickness were analyzed using a Levene test. A one-way ANOVA was used for the homogeneity of variance assumption. On the other hand, Welch's t-test was used for the data of no homogeneity of variance assumption. Moreover, if the results showed a significant difference, they were further analyzed using either Tukey’s test after a one-way ANOVA or Games-Howell tests after Welch’s t-test as a post hoc test. Non-normally distributed data were analyzed using a Kruskal-Wallis test. The significance level was defined as $\mathrm{p}<0.05$.

Results

Characteristic of the subjects

The results of the evaluation of the characteristics of the subjects are shown in Table 1 . There were no significant differences between the groups.

\section{Current intensity and VAS}

The current intensity and VAS results obtained at the fifth min of ES are shown in Table 2. No significant difference was seen in the values between the groups.

\section{Muscle thickness}

Acute changes of infraspinatus muscle thickness are shown in Table 3. The superior infraspinatus muscle thickness was significantly greater after the ES application than the initial thickness measurement in all of the groups, except for the CON group. The inferior infraspinatus muscle thickness of the IR, NEUT, and ER groups was significantly greater after the ES intervention than the initial thickness measurement, however, no significant difference was found in the CONCEN and CON groups between the before and after results. 
The percentage change of muscle thickness for the five groups is shown in Table 4. A one-way ANOVA showed an insignificant difference in the superior infraspinatus results among the groups. Conversely, a significant difference was found for the inferior infraspinatus. In addition, the post hoc test showed that the percentage change in muscle thickness of the inferior infraspinatus for the IR and NEUT groups was significantly higher than the CON group.

\section{Discussion}

We investigated the acute effects of ES on the infraspinatus muscle thickness using different muscle contraction conditions: isometric or concentric. We also investigated the effect of different shoulder positions: maximum internal rotation, neutral rotation, or maximum external rotation. There was no difference in the characteristics of subjects among groups. Furthermore, there were no significant differences among groups in current intensity or VAS. Therefore, we consider that ES were applied to all subjects in a same condition except for types of muscle contractions and shoulder joint positions.

In this study, the percentage change in muscle thickness of the inferior infraspinatus was significantly higher in the IR and NEUT groups than the CON group. The results indicated that performing ES on an isometric contraction with maximum internal and neutral rotations induced muscle swelling immediately after stimulation in the inferior infraspinatus. Fahey et al. ${ }^{15}$ reported that performing ES on the lengthened quadriceps muscle could be more effective than when the muscle is in the shortened position, which is consistent with our results. Maïsetti et al. ${ }^{21}$ reported that muscle tension increased with muscle stretching. Therefore, when the infraspinatus muscle was stretched in the IR group, there might be greater tension during ES than in the CON group, which potentially induced the significant increase in muscle thickness. In this study, muscle swelling of the inferior infraspinatus was found in the NEUT group as well as the IR group. There is a possibility that there was greater tension, because the infraspinatus muscle was stretched, in the NEUT group as well as the IR group. However, we could not measure the muscle tension during the 
ES intervention; therefore, further study is needed to clarify the relationship between muscle swelling and muscle tension during ES intervention.

In contrast, there were no significant differences in the percentage change of muscle thickness of the superior infraspinatus among all groups. Ackland et al. ${ }^{22}$ reported that the external rotation moment arm was $18.1 \mathrm{~mm}$ in the superior infraspinatus, and $18.6 \mathrm{~mm}$ in the inferior infraspinatus, with the shoulder abducted at $90^{\circ}$. The moment arm of the superior infraspinatus was smaller than the inferior infraspinatus, therefore the superior infraspinatus was not stretched by maximum internal rotation and neutral rotation at a $90^{\circ}$ abduction. Thus, it is likely that there was lower tension in the superior infraspinatus than the inferior infraspinatus in the IR and NEUT groups, which may have resulted in the insignificant change in the superior infraspinatus for the IR and NEUT groups.

In addition, muscle thickness of the superior and inferior infraspinatus significantly increased in the IR, NEUT, and ER groups after the ES intervention. Specifically, isometric ES caused immediate muscle swelling after ES. Conversely, muscle thickness of only the superior infraspinatus increased in the CONCEN group. The possible reason for this may be that the electrodes attached to the motor point were off position due to joint movement during the concentric contraction, resulting in insufficient muscle contraction of inferior infraspinatus in the CONCEN group. Therefore, isometric contractions might produce a more effective stimulation of the motor point owing to the stable electrode position.

A limitation of this study is that we did not investigate the association between acute muscle swelling and hypertrophy after the ES application. The correlation between acute muscle swelling and muscle hypertrophy remains undetermined. Further studies are needed to confirm whether ES intervention with maximum internal rotation and neutral rotation can induce hypertrophy and increase strength of the infraspinatus muscle. 


\section{Conclusions}

The results of this study showed that the inferior infraspinatus muscle thickness increased after the ES isometric contraction in maximum internal rotation and neutral rotation of the shoulder. This result suggests that ES is more effective in positions with the muscle stretched. Moreover, this suggests that ES could potentially be an effective method to induce hypertrophy and increase strength of the infraspinatus muscle. This will need to be investigated in future studies. 


\section{REFERENCES}

1. Young SW, Dakic J, Stroia K, Nguyen ML, Harris AH, Safran MR. High Incidence of Infraspinatus Muscle Atrophy in Elite Professional Female Tennis Players. Am J Sports Med. 2015;43(8):1989-1993.

2. Pohlgeers KM, Becker JA. Infraspinatus atrophy in a volleyball player: a case of a bennett lesion causing nerve impingement. Curr Sports Med Rep. 2014;13(6):358-360.

3. Lajtai G, Pfirrmann CW, Aitzetmuller G, Pirkl C, Gerber C, Jost B. The shoulders of professional beach volleyball players: high prevalence of infraspinatus muscle atrophy. Am J Sports Med. 2009;37(7):1375-1383.

4. Ferretti A, Cerullo G, Russo G. Suprascapular neuropathy in volleyball players. J Bone Joint Surg Am. 1987;69(2):260-263.

5. Ferretti A, De Carli A, Fontana M. Injury of the suprascapular nerve at the spinoglenoid notch. The natural history of infraspinatus atrophy in volleyball players. Am J Sports Med. 1998;26(6):759-763.

6. Silinskas V, Grunovas A, Stanislovaitiene J, Buliuolis A, Trinkunas E, Poderys J. Effect Of Electrical Myostimulation On The Function Of Lower Leg Muscles. J Strength Cond Res. 2016.

7. Numata H, Nakase J, Inaki A, et al. Effects of the belt electrode skeletal muscle electrical stimulation system on lower extremity skeletal muscle activity: Evaluation using positron emission tomography. J Orthop Sci. 2016;21(1):53-56.

8. Kibisa R, Grunovas A, Poderys J, Grunoviene D. Restoration of the work capacity of the skeletal muscle with electrical myostimulation. J Strength Cond Res. 2013;27(2):449-457.

9. Gulick DT, Castel JC, Palermo FX, Draper DO. Effect of patterned electrical neuromuscular stimulation on vertical jump in collegiate athletes. Sports Health. 2011;3(2):152-157.

10. Valli P, Boldrini L, Bianchedi D, Brizzi G, Miserocchi G. Effect of low intensity electrical stimulation on quadriceps muscle voluntary maximal strength. J Sports Med Phys Fitness. 2002;42(4):425-430. 
11. Lai HS, Domenico GD, Strauss GR. The effect of different electro-motor stimulation training intensities on strength improvement. Aust J Physiother. 1988;34(3):151-164.

12. Bircan C, Senocak O, Peker O, et al. Efficacy of two forms of electrical stimulation in increasing quadriceps strength: a randomized controlled trial. Clin Rehabil. 2002;16(2):194-199.

13. Filipovic A, Kleinoder H, Dormann U, Mester J. Electromyostimulation--a systematic review of the effects of different electromyostimulation methods on selected strength parameters in trained and elite athletes. J Strength Cond Res. 2012;26(9):2600-2614.

14. Rebai H, Barra V, Laborde A, Bonny JM, Poumarat G, Coudert J. Effects of two electrical stimulation frequencies in thigh muscle after knee surgery. Int J Sports Med. 2002;23(8):604-609. 15. Fahey TD, Harvey M, Schroeder RV, Ferguson F. Influence of sex differences and knee joint position on electrical stimulation-modulated strength increases. Med Sci Sports Exerc. 1985;17(1):144-147.

16. Yasuda T, Fukumura K, Iida H, Nakajima T. Effect of low-load resistance exercise with and without blood flow restriction to volitional fatigue on muscle swelling. Eur J Appl Physiol. 2015;115(5):919-926.

17. Farup J, de Paoli F, Bjerg K, Riis S, Ringgard S, Vissing K. Blood flow restricted and traditional resistance training performed to fatigue produce equal muscle hypertrophy. Scand J Med Sci Sports. 2015;25(6):754-763.

18. Dangott B, Schultz E, Mozdziak PE. Dietary creatine monohydrate supplementation increases satellite cell mitotic activity during compensatory hypertrophy. Int J Sports Med. 2000;21(1):13-16.

19. Fahs CA, Loenneke JP, Thiebaud RS, et al. Muscular adaptations to fatiguing exercise with and without blood flow restriction. Clin Physiol Funct Imaging. 2015;35(3):167-176.

20. Hasegawa S, Kobayashi M, Arai R, Tamaki A, Nakamura T, Moritani T. Effect of early implementation of electrical muscle stimulation to prevent muscle atrophy and weakness in patients after anterior cruciate ligament reconstruction. J Electromyogr Kinesiol. 2011;21(4):622-630. 
21. Maisetti O, Hug F, Bouillard K, Nordez A. Characterization of passive elastic properties of the human medial gastrocnemius muscle belly using supersonic shear imaging. J Biomech. 2012;45(6):978-984.

22. Ackland DC, Pandy MG. Moment arms of the shoulder muscles during axial rotation. J Orthop Res. 2011;29(5):658-667. 
Authors' contributions. -

Ko Yanase designed and performed both the experiment and the statistical analysis, and drafted the manuscript. Satoshi Hasegawa conceived the study and helped draft the manuscript. Masatoshi Nakamura conceived the study and helped draft the manuscript. Hiroki Tanaka conceived the study and helped to perform the experiment. Jun Umehara conceived the study and helped to perform the experiment. Kojiro Araki helped to perform the experiment and perform the statistical analysis. Satoko Ibuki helped to draft the manuscript. Noriaki Ichihashi conceived the study and helped to draft the manuscript.

Funding. - Nothing

Conflicts of interest. - All of the authors declare that they have no competing interests in the study.

Acknowledgements. - The authors would like to thank all the participants of this study. 


\section{TITLES OF TABLES}

Table I. - General characteristic of the subjects

Table II. - Current intensity and VAS

Table III. - Initial and final muscle thickness

Table IV. - Percentage change in muscle thickness of the infraspinatus muscle

\section{TITLES OF FIGURES}

Figure 1. - Area of muscle thickness measurement

(1) Superior Infraspinatus, (2)Inferior infraspinatus

Figure 2. - Different positions and types of contraction

(1) Isometric contraction with maximum internal rotation (IR)

(2) Isometric contraction with neutral position of the rotation (NEUT)

(3) Isometric contraction with maximum external rotation (ER)

(4) Concentric contraction (CONCEN) 
Table I .-General characteristic of the subjects

\begin{tabular}{ccccccc}
\hline & CON & IR & NEUT & ER & CONCEN & \\
& $(\mathrm{n}=8)$ & $(\mathrm{n}=8)$ & $(\mathrm{n}=8)$ & $(\mathrm{n}=8)$ & $(\mathrm{n}=8)$ & p-value \\
\hline Age (years) & $23.8 \pm 3.5$ & $25.0 \pm 5.3$ & $23.9 \pm 2.5$ & $25.0 \pm 4.5$ & $24.1 \pm 1.7$ & 0.884 \\
Body mass (kg) & $172.3 \pm 6.4$ & $173.1 \pm 6.6$ & $170.9 \pm 3.5$ & $171.1 \pm 6.3$ & $173.0 \pm 5.8$ & 0.898 \\
Body height (cm) & $64.4 \pm 8.1$ & $64.0 \pm 5.0$ & $71.5 \pm 6.4$ & $66.8 \pm 7.2$ & $70.1 \pm 9.4$ & 0.128 \\
\hline
\end{tabular}

Mean \pm SD (Standard deviation) 
Table II .- Current intensity and VAS

\begin{tabular}{cccccc}
\hline & IR & NEUT & ER & CONCEN & p-value \\
& & & & & \\
\hline Current Intensity (mA) & $83.5 \pm 20.4$ & $85.0 \pm 27.2$ & $79.0 \pm 20.4$ & $86.5 \pm 27.2$ & 0.292 \\
VAS (mm) & $63.1 \pm 19.0$ & $65.9 \pm 18.8$ & $59.8 \pm 19.9$ & $49.4 \pm 13.6$ & 0.475 \\
\hline
\end{tabular}

Mean \pm SD (Standard deviation) 
Table III.-Initial and final muscle thickness

\begin{tabular}{|c|c|c|c|c|c|c|}
\hline \multirow[t]{2}{*}{ Thickness(cm) } & \multicolumn{2}{|c|}{ Superior infraspinatus } & \multicolumn{4}{|c|}{ Inferior infraspinatus } \\
\hline & Initial & Final & & Initial & Final & \\
\hline CON & $1.05 \pm 0.12$ & $1.09 \pm 0.13$ & 0.063 & $1.38 \pm 0.33$ & $1.39 \pm 0.33$ & 0.656 \\
\hline IR & $0.96 \pm 0.24$ & $1.05 \pm 0.27^{*}$ & 0.017 & $1.50 \pm 0.37$ & $1.60 \pm 0.38 *$ & 0.003 \\
\hline NEUT & $1.04 \pm 0.11$ & $1.12 \pm 0.14^{*}$ & 0.012 & $1.45 \pm 0.25$ & $1.55 \pm 0.27^{*}$ & 0.000 \\
\hline ER & $1.01 \pm 0.14$ & $1.09 \pm 0.13^{*}$ & 0.002 & $1.58 \pm 0.30$ & $1.67 \pm 0.38^{*}$ & 0.006 \\
\hline CONCEN & $1.13 \pm 0.28$ & $1.21 \pm 0.30 *$ & 0.007 & $1.52 \pm 0.45$ & $1.59 \pm 0.46$ & 0.108 \\
\hline
\end{tabular}

Mean \pm SD (Standard deviation)

* Final measurement significantly greater than the initial measurement $(\mathrm{p}<0.05)$ 
Table IV.-Percentage change in muscle thickness of the infraspinatus muscle

\begin{tabular}{lcccccc}
\hline & CON & IR & NEUT & ER & CONCEN & p-value \\
\hline Superior infraspinatus (\%) & $3.22 \pm 4.46$ & $9.52 \pm 7.89$ & $7.41 \pm 5.48$ & $8.40 \pm 5.24$ & $7.06 \pm 5.37$ & 0.261 \\
Inferior infraspinatus (\%) & $0.52 \pm 3.25$ & $6.49 \pm 2.86^{*}$ & $6.63 \pm 4.76^{*}$ & $5.25 \pm 3.28$ & $4.33 \pm 5.84$ & 0.026 \\
\hline
\end{tabular}

Mean \pm SD (Standard deviation)

* Significant difference between the representative group and the CON group $(\mathrm{p}<0.05)$ 


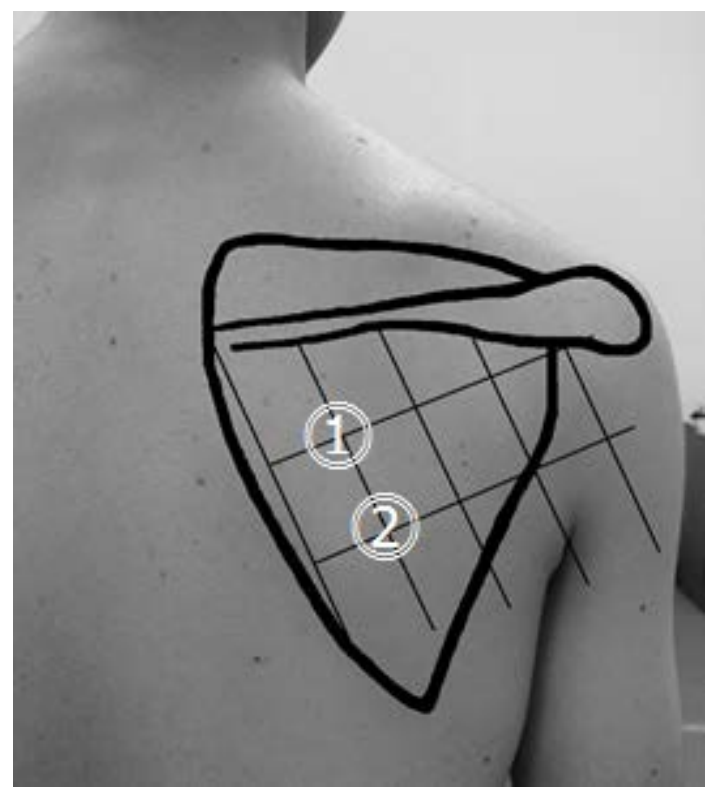

Figure 1 Area of muscle thickness measurement

(1) Superior Infraspinatus, (2)Inferior infraspinatus 


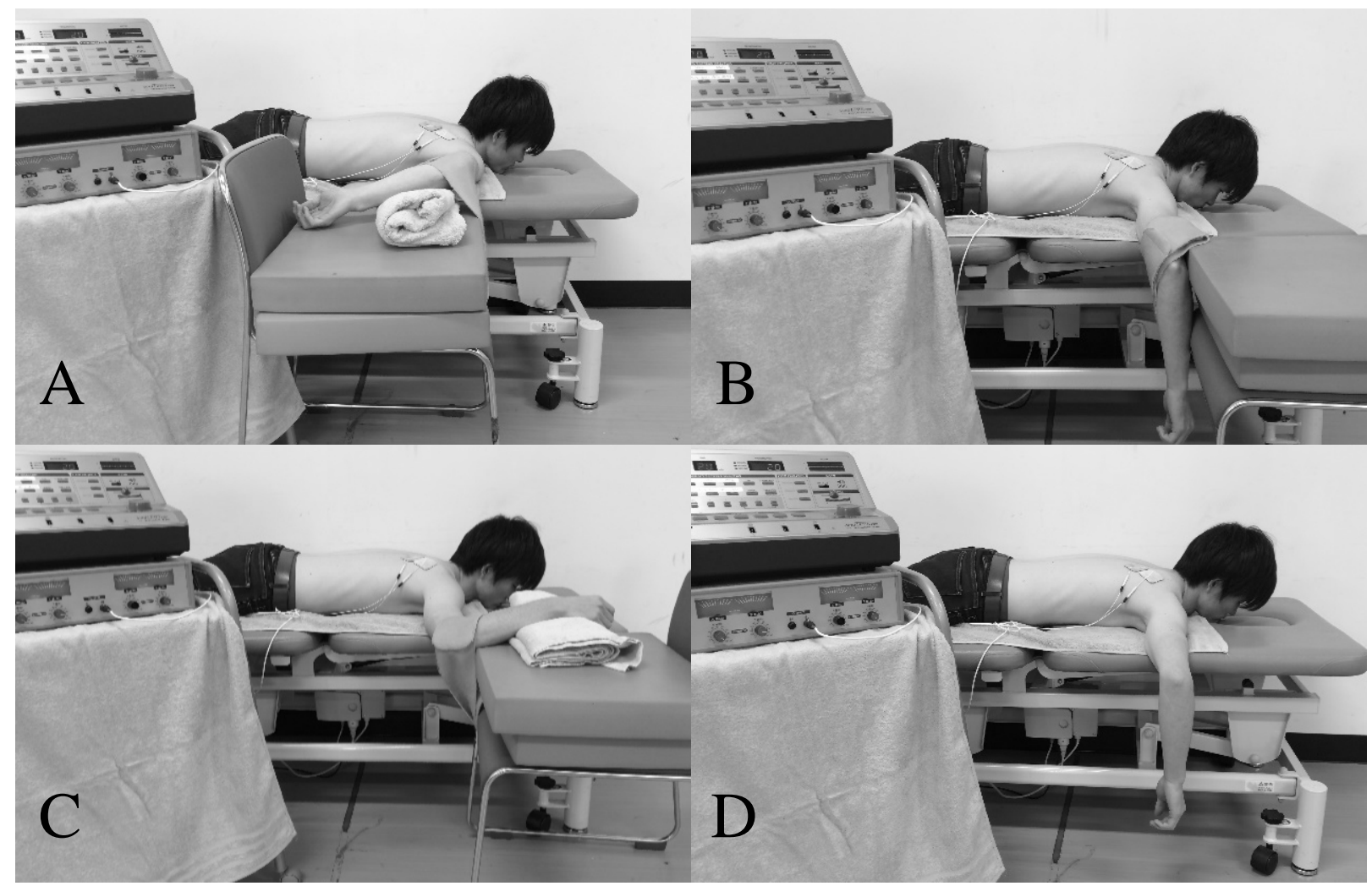

Figure 2 Different positions and types of contraction
A) Isometric contraction with maximum internal rotation (IR)
B) Isometric contraction with neutral position of the rotation (NEUT)
C) Isometric contraction with maximum external rotation (ER)
D) Concentric contraction (CONCEN) 